\title{
Review on Color Image Edge Detection using Quaternion Fourier transform
}

\author{
Jill A. Patel ${ }^{1}$, Minkal B. Patel ${ }^{2}$ \\ ${ }^{1,2}$ Silver Oak College of Engineering and Technology, Ahmedabad, Gujarat, India
}

\begin{abstract}
Edge of the image is an elementary step in various images processing application. The problem arising in the edge detection are poor edge localization, less noise capacity, unable to detect edges in complex background images and inability to properly detect the color edges in image. This paper proposes several techniques used to overcome the existing problems. Moreover it also shows the detail description of the novel approach called Quaternion Fourier Transform for edge detection.
\end{abstract}

Keywords: Hyper Complex Numbers, Quaternion Fourier Transform (QFT), Image Edge Detection, Image Processing, Sobel, Prewitt, And Fuzzy.

\section{Introduction}

Image processing is a method to convert an image into digital form and some operations are performed on it, in order to get an Enhanced image or to extract some useful information from it. Image processing is used in various applications like image enhancement, removal of noise, image classification, image edge detection and many more.

In digital image, the edge is a collection of pixels whose gray value has a step or roof change, and refers to the part where the brightness of the image local area changes significantly. The gray profile in this region can generally be seen as a step i.e. in a small buffer area, a gray value rapidly changes to another whose gray value is largely different with it. Edge widely exists between objects and backgrounds, objects and objects, primitives and primitives. The edge of an object is reflected in the discontinuity of the gray. Therefore, the general method of edge detection is to study the changes of a single image pixel in gray area, use the variation of the edge neighboring first order or second order to detect the edge. ${ }^{[14]}$ This method is used to refer as local operator edge detection method. Edge detection is mainly the measurement, detection and location of the changes in gray image. Image edge is the most basic feature of the image. When we observe the objects, the clearest part we see firstly is edge and line. According to the composition of the edge and line, the object structure can be known. Therefore, edge extraction is an important technique in graphics processing and feature extraction.

The basic idea of edge detection is as follows: - First, use edge enhancement operator to highlight the local edge of the image. Then, define the pixel "edge strength" and set the threshold to extract the edge point set. ${ }^{[14]}$ Because of the noise and blurring image, the edge detected may not be continuous. So, edge detection includes two contents first is using edge operator to extract the edge point set. Second is removing some of the edge points from the edge point set, filling it with some another and linking the obtained edge point set into lines.

The paper aims to describe proposed method in detail Section 2 shows the survey done to know the work done and history. Section 3 shows different techniques for image edge detection. Section 4 describes proposed method in detail.

\section{Literature Survey}

It shows the Relationship between image and its counterpart using 2D-QFT. It explains the properties of QFT spectrum. Limitation of FT as compared to QFT, and shows the applicability of QFT to color image. ${ }^{[1]}$

It shows comparison of different techniques, Flaws existing in the image edge detection, Represent the four different, image edge detection techniques, Image edge detection based on wavelet transform, mathematics morphology, fuzzy and fractal geometry. ${ }^{[2]}$

It shows the use of edge detection is ctscan images, Reasons for selecting the gradient of weibull distribution, Edge detection using gradient of Weibull distribution and Contribution of edge detection in medical domain. ${ }^{[3]}$

It shows Flaw in the prevailing edge detection techniques, Hybrid approach is proposed to overcome the limitation, Description of the hybrid approach and Comparison among sequential and parallel edge detection is shown. ${ }^{[4]}$

\section{Different Techniques for Edge Detection}




\section{International Journal of Science and Research (IJSR) \\ ISSN (Online): 2319-7064}

Index Copernicus Value (2013): 6.14 | Impact Factor (2014): 5.611

\begin{tabular}{|c|c|c|c|}
\hline Techniques & Description & Advantages & Disadvantages \\
\hline Sobel & $\begin{array}{l}\text { Sobel filter is used in image processing and computer vision, } \\
\text { particularly within edge detection algorithms where it creates } \\
\text { an image emphasizing edges. It is a discrete differentiation } \\
\text { operator, computing an approximation of the gradient of the } \\
\text { image intensity function. }\end{array}$ & $\begin{array}{l}\text { Simplicity, Detection of } \\
\text { edges and their orientations, } \\
\text { place emphasis on pixel }\end{array}$ & Sensitive to noise, Inaccurate \\
\hline Prewitt & $\begin{array}{l}\text { The Prewitt operator is used in image processing, particularly } \\
\text { within edge detection algorithms. At each point in the } \\
\text { image, the result of the Prewitt operator is either the } \\
\text { corresponding gradient vector or the norm of this vector. }\end{array}$ & \begin{tabular}{|c|} 
Simplicity, Detection of \\
edges and their Orientation
\end{tabular} & $\begin{array}{l}\text { Sensitive to noise, Inaccurate } \\
\text { does not emphasis on pixel }\end{array}$ \\
\hline Fuzzy & $\begin{array}{l}\text { A fuzzy rule base algorithm which is capable of detecting } \\
\text { edges efficiently from the gray scale images. }\end{array}$ & $\begin{array}{l}\text { Efficiently handles } \\
\text { uncertainty }\end{array}$ & $\begin{array}{l}\text { Requires predefined database, } \\
\text { Inefficient for noisy images }\end{array}$ \\
\hline
\end{tabular}

\section{Quaternion Fourier Transform}

The Fourier transforms plays a critical role in broad range of image processing applications, including enhancement, restoration, analysis and compression. For filtering of gray scale images 2D Fourier transform is an important tool.

This converts the image from spatial domain to frequency domain and then by applying filtering mask filtering is done. To filter color an image, a new approach is implemented which uses hyper complex numbers (called as Quaternion) to represent color images and uses Quaternion Fourier Transform for filtering. This transform allows color images to be transformed as whole, rather than as color separated component.

The Quaternion Fourier Transform is well suited for describing the spectral content of color images. Similar to gray scale images, color images represented as quaternion valued images can also be transformed into the Frequency domain and can be represented as quaternion frequency signals, based on which different image processing techniques such as filtering can be performed efficiently. Filtering in quaternion frequency domain has advantage that the color triples are processed as a whole unit rather than dealing with RGB channels separately. We believe more accurate color information can be preserved this way, since all color channels are processed as a single unit. And would like to point out that, the Quaternion Fourier Transform is not limited to this, but also can be applied to other color image processing fields, such as image registration, edge detection, and data compression.

Based on the concept of quaternion multiplication and exponential, the Discrete Quaternion Fourier Transform (DQFT) has been introduced. Due to non-commutative properly of the quaternion, there are three different types of DQFT defined: the left side DQFT, the right side DQFT and the two sides DFQT represented as,

Two-sided DQFT (Type1)

$$
F_{L-R}(u, v)=\frac{1}{\sqrt{M N}} \sum_{x=0}^{M-1} \sum_{y=0}^{N-1} \boldsymbol{e}^{-2 \pi \mu \frac{x \mu}{M}} f(x, y) e^{-2 \mu \pi \frac{v y}{N}}
$$

Left-sided DQFT (Type2)

$F_{L}(u, v)=\frac{1}{\sqrt{M N}} \sum_{x=0}^{M-1} \sum_{y=0}^{N-1} e^{-2 \pi \mu\left(\frac{x \mu}{M}+\frac{y v}{N}\right)} f(x, y)$
Right-sided DQFT (Type-3)

$F_{R}(u, v)=\frac{1}{\sqrt{M N}} \sum_{x=0}^{M-1 N-1} \sum_{y=0}^{N-1} f(x, y) e^{-2 \pi u\left(\frac{x \mu}{M}+\frac{y v}{N}\right)}$

\section{Results and Discussions}

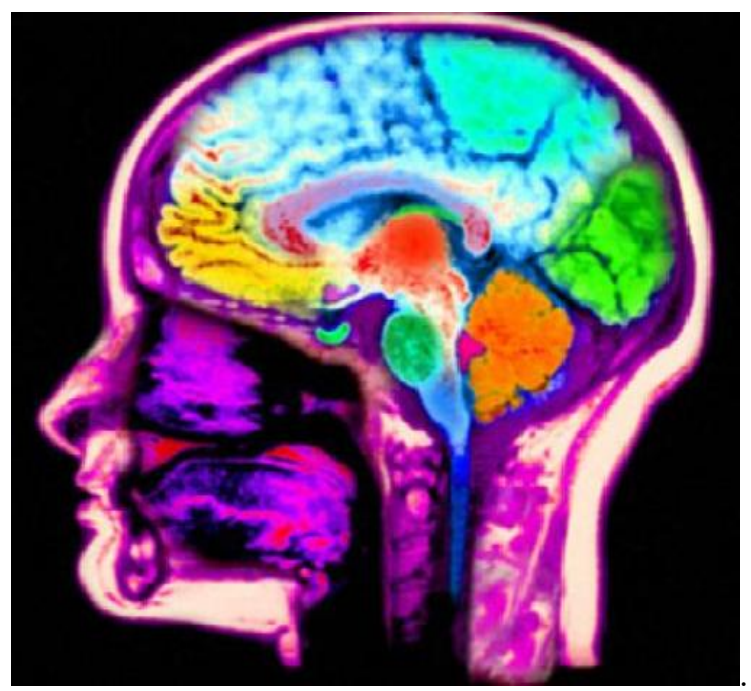

Figure 1: Original image
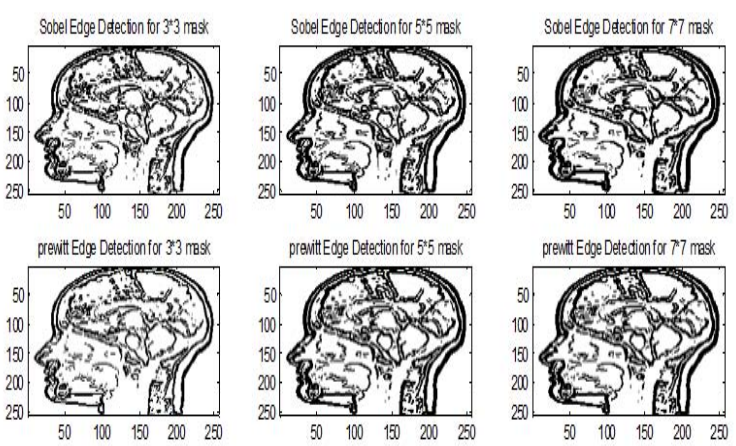

prewitt Edge Dededionfor 77 mas
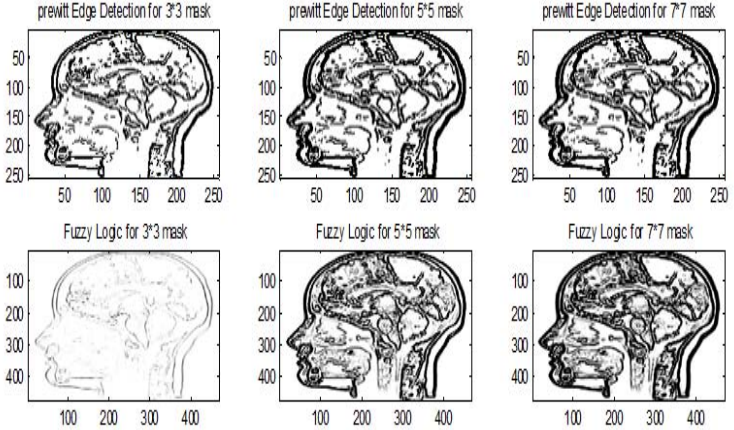

Firzy Logic fo $7^{77} 7_{\text {mesk }}$

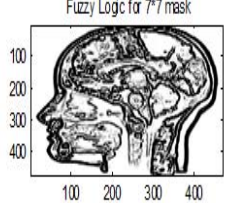

Figure 2: Edge Detection 


\section{International Journal of Science and Research (IJSR) \\ ISSN (Online): 2319-7064}

Index Copernicus Value (2013): 6.14 | Impact Factor (2014): 5.611

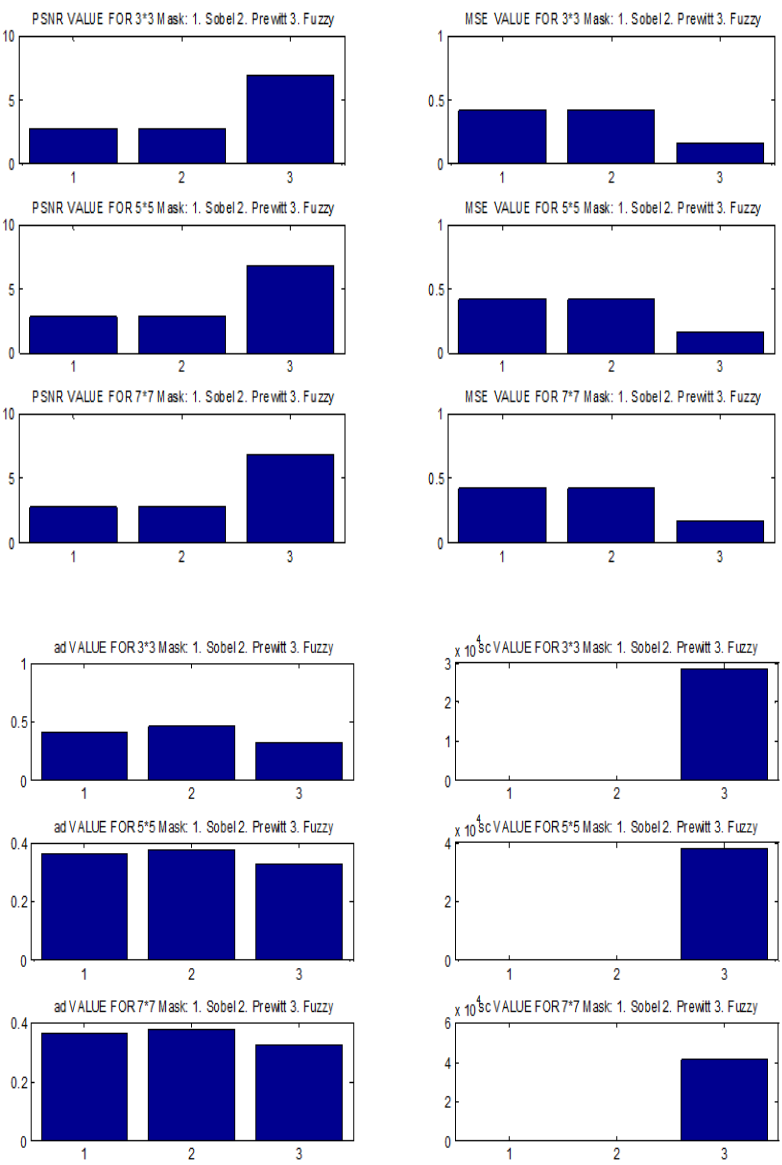

Figure 3: Comparison of parameters

\section{Conclusion}

Image detection has been developed for gray scale images till date. The proposed paper helps to understand the different techniques existing in the edge detection and comparison is made between the existing techniques. The proposed paper also shows the novel approach in edge detection that is quaternion transform, which is useful to process the complete unit of RGB images as a single unit.

\section{References}

[1] Zheng Lu1, Yi Xu1, Xiaokang Yang1, Li Song1 and Leonardo Traversoni “2D Quaternion Fourier Transform: The Spectrum Properties and its Application in Color Image Registration” 2007 IEEE.

[2] Lei Zhai, Shouping Dong, Honglian Ma "Recent Methods and Applications on Image Edge Detection" 2008 IEEE

[3] Wafaa Kamel, Al-Jibroy, Ali El-Zaart "A New Edge Detector in CT Scan Images using Gradient of Weibull Distribution" Vol. 4, No. 1, 13-17, 2014 (WCSIT).

[4] Chinu, Amit Chhabra "Hybrid Approach for Color based Image Edge Detection" 2014 IEEE

[5] Vikas R. Dubey "Quaternion Fourier Transform for Colour Images" (IJCSIT) 2014.

[6] B.D.Venkatramana Reddy1 and Dr.T.Jayachandra Prasad2 "Colour-Texture Image Segmentation using Hyper complex Gabor Analysis”(SIPIJ) Vol.1, No.2, December 2010.
[7] Min-Hung Yeh "Relationships among various 2-D Quaternion Fourier Transforms"

[8] Mawardi Bahri1, Ryuichi Ashino, Remi Vaillancourt3 "Two-Dimensional Quaternion Fourier Transform of type ii and Quaternion Wavelet Transform" 2012 IEEE.

[9] Ramya R, Dr. Srinivasa Babu P "Automatic Tuberculosis Screening Using Canny Edge Detection Method" 2015 IEEE

[10]Libao Zhang and Kaina Yangs "Region-of-Interest Extraction Based on Frequency Domain Analysis and Salient Region Detection for Remote Sensing Image" 2013 IEEE.

[11]B.D.Venkatramana Reddy1, Dr.T.Jayachandra Prasad2"Frequency Domain Filtering of Colour Images using Quaternion Fourier Transforms" IJCST Vol. 1, Iss ue 2, December 2010.

[12]G.T. Shrivakshan"A Comparison of various Edge Detection Techniques used in Image Processing" IJCSI Vol. 9, Issue 5, No 1, September 2012.

[13] Raman Mani, Dr. Himanshu Aggarwal "Study and comparison of Various Image edge Detection Techniques" (IJIP) Vol (3)

[14] Wenshuo Gao, Lei Yang, Xiao Guang, Zhang Huizhong Liu. "An improved sobel edge detection". 978-1-42445540-9/10/\$26.00 (C2010 IEEE.

\section{Author Profile}

Jill A. Patel, Student of M.E. (Electronics \& Communication) final year at Gujarat Technological University, Ahmedabad, India from Silver Oak College of Engineering \& Technology.

Minkal B. Patel, M.E (Electronics \& Communication) working as Asst professor at Silver Oak College of Engineering \& Technology, Ahmedabad India. 